\title{
Dynamical Mechanism of Circadian Singularity Behavior in
}

\section{Neurospora}

\author{
Maorong Sun ${ }^{1}$, Yi wang ${ }^{2}$, Xin $\mathrm{Xu}^{2}$ and Ling Yang ${ }^{2,3, *}$
}

1. Faculty of Science, Jiangsu University, Zhenjiang 212013,China

2. School of Mathematical Sciences, Soochow University, Suzhou 215006, China

3. Center for Systems Biology, Soochow University, Suzhou 215006, China

* Corresponding author: Ling Yang, E-mail address: lyang@suda.edu.cn

\begin{abstract}
Many organisms have oscillators with a period of about 24 hours, called "circadian clocks”. They employ negative biochemical feedback loops that are self-contained within a single cell (requiring no cell-to-cell interaction). Circadian singularity behavior is a phenomenon of the abolishment of circadian rhythmicities by a critical stimulus. These behaviors have been found experimentally in Neurospora, human and hamster, by temperature step-up or light pulse. Two alternative models have been proposed to explain this phenomenon: desynchronization of cell populations, and loss of oscillations in all cells by resetting the each cell close to a steady state. In this work, we use a mathematical model to investigate the dynamical mechanism of circadian singularity behavior in Neurospora. Our findings suggest that the arrhythmic behavior after the critical stimulus is caused by the collaboration of the desynchronization and the loss of oscillation amplitude. More importantly, we found that the stable manifold of the unstable equilibrium point, instead of the steady state itself, plays a crucial role in circadian singularity behavior.
\end{abstract}

Key words: circadian clock, singularity behavior, desynchronization, steady state, stable manifold 


\section{Introduction}

Most organisms have the capability of adapting to their periodically changing environment by exhibiting rhythms of periodicity of approximately $24 \mathrm{~h}$ [1-3]. Such circadian rhythms are endogenous as they generally persist in constant conditions (without environmental change, like light/dark switch). The central mechanism of the circadian oscillations is a negative feedback loop: there are positive elements that activate clock genes that yield clock proteins that act in some way to block the activity of the positive elements [4-6]. The changes conferring altered circadian period, phase or amplitude are associated with physiological disorders and decreased lifespan [7-9].

One of the most mysterious phenomena of circadian clocks is that the rhythmicities can be abolished after a proper stimulus (at an appropriate time with the right strength), such as light or temperature change. This phenomenon is called singularity behavior. The abolishment of the rhythmicities has been observed in different species, including Human[10], mice[11], Neurospora[12] and hamster[13]. Ukai et. al [11] synthetically produced photo-responsiveness within mammalian cells and continuously monitored the effect of photo-perturbation on the state of cellular clocks. In their experiment, when the cells are exposed to a critical light pulse, the phases of cells are abruptly diversified by the pulse, suggesting that desynchronization was induced by the light pulse. Therefore, these results indicates that desynchronization of individual cellular clocks underlies singularity behavior. Huang et. al [12] demonstrated that both temperature step up (from 21C to 25C) and light pulse (15 seconds), can trigger singularity behavior in Neurospora. However, when they mixed the cultures that were treated separately with a 19-25C temperature shift or a 22.5-25C step up around CT17 (the anti-phase groups), robust rhythmicity was observed immediately afterwards and no singularity-like behavior was seen. Thus, they don't think that the singularity behavior in Neurospora is solely caused by desynchronization of the population of oscillators. Therefore, they proposed that after the critical stimulus, all cells are at or close to the steady state and the amplitudes of them are close to zero [12, 14].

Mathematical models have been used to account for the suppression of circadian rhythms [14-16]. In a 2-variable limit cycle model, the circadian oscillator cycles with its preferred amplitude in constant conditions. When a critical stimulus is applied, the clock can then be sent to the singularity point (unstable center), where the clock becomes phaseless and arrhythmic $[15,16]$. Leloup and Goldbeter developed a biochemical model to explan the long-term suppression of circadian rhythms [14]. In their model, a stable limit cycle coexists with a stable steady state; these 2 attractors are separated by an unstable limit cycle. The pulse-triggered transition of pacemaker cells from a stable oscillatory regime into a stable steady state.

Because negative feedback loop is a common prerequisite in many circadian oscillations, we use a simple negative feedback model developed by Gonze et. al. [13] for Neurospora, as a example, to test these two alternative explanations of the phenomenon (desynchronization of the phases or loss of oscillation amplitude in all cells).

\section{Mathematical model}

We used the minimal model developed by Gonze and colleagues [13] to study the singularity behavior in Neurospora. As schematized in figure 1.A, the protein FRQ, producted of the frq gene, migrates to the nucleus where it represses the transcription of frq gene itself; light 
controls the circadian system by inducing the transcription of frq. The theoretical model presented below for circadian oscillations in Neurospora incorporates the negative autoregulatory feedback
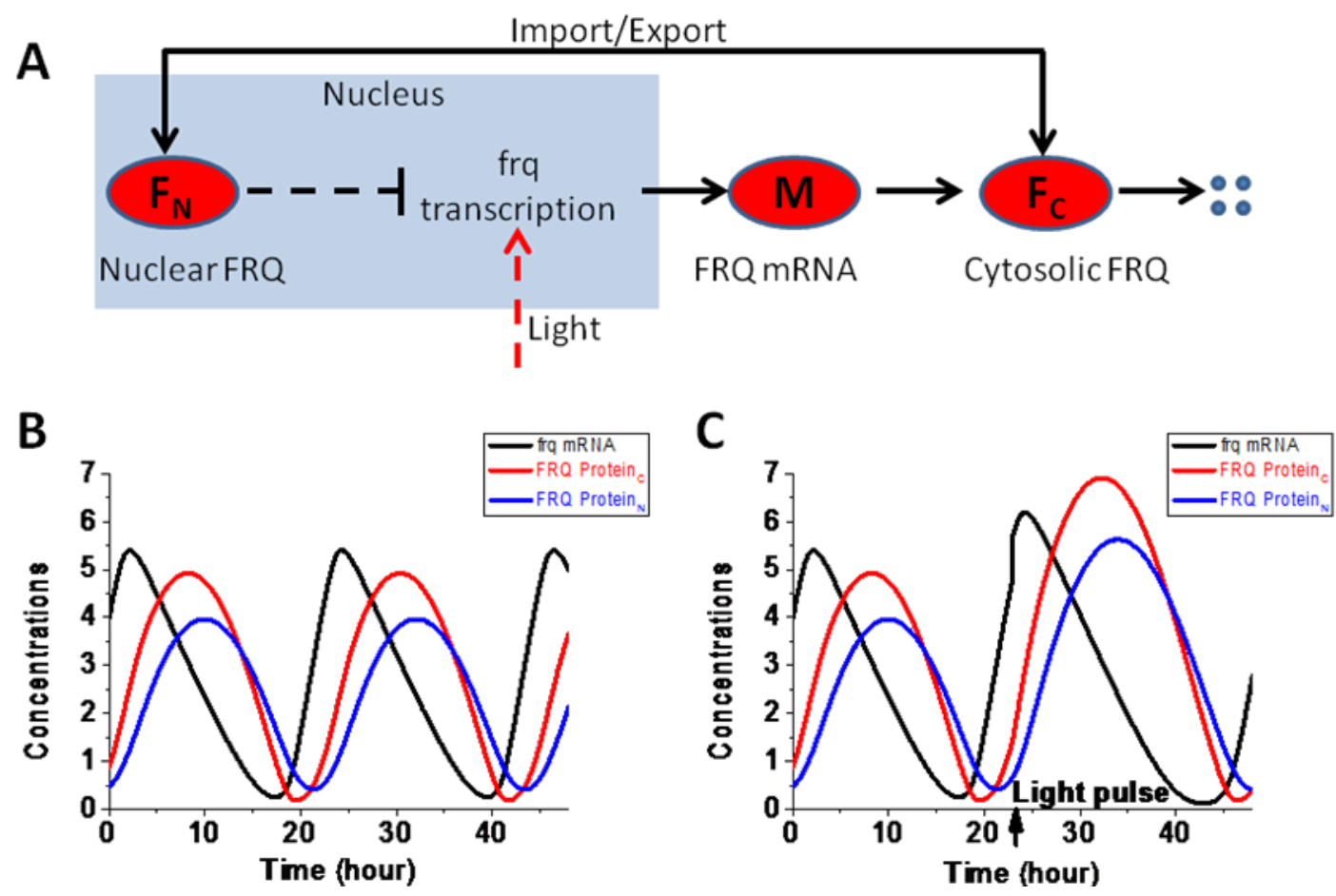

loop involving FRQ, as well as the specific effects of light in the system.

The minimal model for circadian oscillations of FRQ and frq mRNA in Neurospora is then governed by the following set of three kinetic equations:

$$
\left\{\begin{array}{l}
\frac{\mathrm{dM}}{\mathrm{dt}}=\mathrm{vS} * \frac{\mathrm{K}_{1}^{\mathrm{n}}}{\mathrm{K}_{1}^{\mathrm{n}}+\mathrm{F}_{\mathrm{N}}^{\mathrm{n}}}-\mathrm{vm} \frac{\mathrm{M}}{\mathrm{k}_{\mathrm{m}}+\mathrm{M}}+\mathrm{I}_{\mathrm{light}} \\
\frac{\mathrm{dF} \mathrm{F}_{\mathrm{c}}}{\mathrm{dt}}=\mathrm{ks} * \mathrm{M}-\mathrm{vd} * \frac{\mathrm{F}_{\mathrm{c}}}{\mathrm{K}_{\mathrm{d}}+\mathrm{F}_{\mathrm{c}}}-\mathrm{k}_{1} * \mathrm{~F}_{\mathrm{c}}+\mathrm{k}_{2} * \mathrm{~F}_{\mathrm{N}} \quad \text { (1)cdi } \\
\frac{\mathrm{d} \mathrm{F}_{\mathrm{N}}}{\mathrm{dt}}=\mathrm{k}_{1} * \mathrm{~F}_{\mathrm{c}}-\mathrm{k}_{2} * \mathrm{~F}_{\mathrm{N}}
\end{array}\right.
$$

In these equations, the three variables $\mathrm{M}, \mathrm{F}_{\mathrm{C}}$ and $\mathrm{F}_{\mathrm{N}}$ denote, respectively, the concentrations (defined with respect to the total cell volume) of the frq mRNA and of the cytosolic and nuclear forms of FRQ. According to reference[17], the light increases frq mRNA synthesis by promoting the DNA transcriptional efficiency. Thus, a light input term is added to the equation of mRNA as $\mathrm{I}_{\text {light. }}$.

This system produces autonomous oscillations (Figure 1.B) of a period close to $21.5 \mathrm{~h}$ in constant conditions, as observed in the experiments. With a 5 second light pulse added at $23 \mathrm{~h}$, a jumping up can be observed in the curve of frq mRNA. The peaks of all molecular concentrations in the second period are increased comparing to the first period. Also, the phases of all curves are delayed. Thus, the light pulse does change the dynamics of the circadian system.

Although the singularity behavior has been observed in experiments[12], we still need to reproduce it in this model, before we investigate its dynamical mechanism. However, the singularity behavior is a small probability event. It can be induced only by the stimulus at the 
appropriate phases and with the right strengths. Therefore, we now focus on reproducing the abolishment of the rhythmicity, and the corresponding stimulus phases/strengths.

\section{Results}

\subsection{The stimulus phase and strength for singularity behavior}

In order to find the right stimulus phase and strength for singularity behavior, we have to scan a two dimensional space (phase/ strength) and measure their output amplitudes. Since singularity behavior is a small probability event, we designed a protocol to reduce the computational time:

1. We assume that the cells run along the stable limit cycle with slight differences due to the balance of cell-cell synchronization and the environmental (or endogenous) disturbance. Thus, we observe the orbits started from a group of slightly different cells, to see if their group outputs (average of the orbits) lost amplitude after the stimulus. The cells in the group are selected around a "central" cell in the stable limit cycle.

2. Roughly scan the stimulus phase and strength, to find an "approximate proper" stimulus which furthest suppresses the rhythmicity.

3. Re-execute the scan close to the "approximate proper" stimulus, to find the more accurate stimulus phases and strengths for singularity behavior.

Based on this protocol, we perform the simulations to look for the small range of the stimuli for singularity behavior.

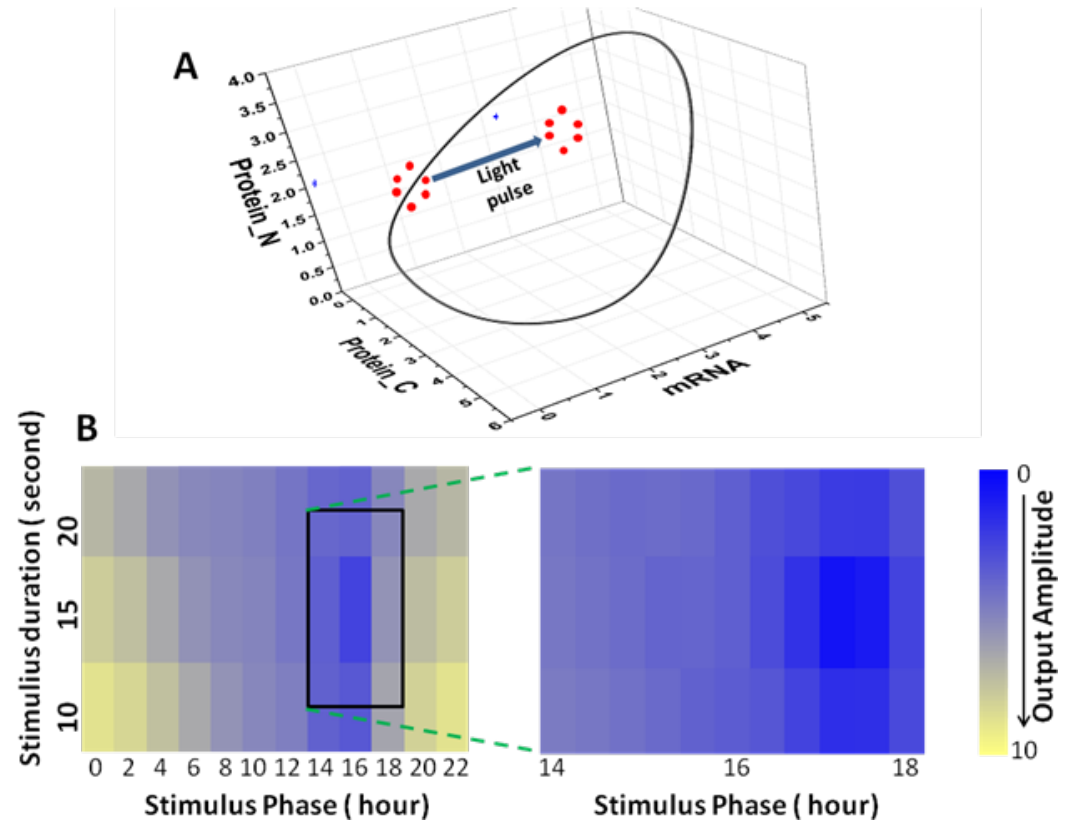

First, we use $\mathrm{M}=3.9796, \mathrm{~F}_{\mathrm{C}}=0.8867, \mathrm{~F}_{\mathrm{N}}=0.4771$, as the initial values at circadian time zero hour ( CT0). Under this initial condition, FRQ protein reaches its trough at CT0, which is consistent with the experimental observation (Figure $1 \mathrm{~B}$ ).

Then, at the phases of each 2 hours (from CT0 hour to CT22 hour), we give light stimuli with three different strengths (light pulse durations: 10s, 15s and 20s). Thus, there are totally $12 * 3$ stimuli. For each stimulus, we test the group output (average of the orbits). We use the point ( $\mathrm{M}^{*}$, $\left.\mathrm{F}_{\mathrm{C}}{ }^{*}, \mathrm{~F}_{\mathrm{N}}{ }^{*}\right)$ in the limit cycle as a central cell, and select six slightly different cells one by one, 
through positively/negatively changing one variable (total three variables) of it. Here we observe the orbits beginning with $\left(\mathrm{M}^{*} \pm 0.1, \mathrm{~F}_{\mathrm{C}}^{*}, \mathrm{~F}_{\mathrm{N}}^{*}\right), \quad\left(\mathrm{M}^{*}, \mathrm{~F}_{\mathrm{C}}{ }^{*} \pm 0.1, \mathrm{~F}_{\mathrm{N}}^{*}\right)$ and $\left(\mathrm{M}^{*}, \mathrm{~F}_{\mathrm{C}} * \mathrm{~F}_{\mathrm{N}}{ }^{*} \pm 0.1\right)$. Figure 2A illustrates the cell selection in the group and the effect of the light pulse stimulus.

Hot map Figure 2B is used to describe the amplitude level in the next 24 hour after the stimulus. The horizontal axis of Fig 2B refers to the stimulus phase, and the longitudinal axis means the stimulus duration. The color means the amplitude level in the next 24 hour after the stimulus. The color bar in the right side of Figure 2B indicates the amplitude level: from zero (blue) to 10 (yellow), in arbitrary unit. The left panel shows the result of the rough scan including (12phases X 3 strengths) stimuli. It reveals that most stimuli maintain the oscillation level in the next 24h , with the amplitudes greater than 3 (half amplitude of that under normal conditions). Hence, most stimuli cannot effectively suppress the oscillation. The suppression of circadian rhythm happens in the areas close to the phase of CT16, with 15 seconds light pulse.

Therefore, we perform a refined scan in the area indicated by a black frame: stimulus phase from CT14 to CT CT18, increasing by 0.4 hour; light pulse durations are $12.5 \mathrm{~s}, 15 \mathrm{~s}$ and $17.5 \mathrm{~s}$. The result shows that the most effective suppression (singularity behavior) occurs at phase 17.2 and light pulse duration 15s. The amplitude under this stimulus is 0.7754 , which is almost the $1 / 7$ of the normal oscillation level. The neighbour of this stimulus, phase 17.4 and light pulse duration 15s, also has a very low amplitude 1.0237 .

Therefore, the simulations conformed that the suppression of circadian rhythm can be certainly induced by a critical light pulse in this model. Furthermore, these simulation results are roughly consistent with the experimental singularity conditions[12]: 15s of light pulse (100 lux) at CT19.

To clarify the dynamical mechanism of singularity behavior and test two alternative hypotheses proposed for the phenomenon, we then observed the detailed responses to a singularity stimulus and other stimuli.

\subsection{The amplitude loss and phase desynchronization}

There are two proposed hypotheses to explain the mechanism of singularity behavior: amplitude loss in all cells or desynchronization in phases. The experimental data from Liu and colleagues [12] supported that after the critical stimulus, all cells are at or close to a steady state and the amplitudes are close to zero; However, Ueda and colleagues provided evidence of strong desynchronization in phases[11].

We now study how cells respond to a singularity stimulus or other stimuli. Figure 3A shows the responses of six slightly different cells (black lines) to a stimulus at CT4 with duration 20s.

The six slightly different cells are selected as $\left(\mathrm{M}^{*} \pm 0.1, \mathrm{~F}_{\mathrm{C}} *, \mathrm{~F}_{\mathrm{N}} *\right), \quad\left(\mathrm{M}^{*}, \mathrm{~F}_{\mathrm{C}} * \pm 0.1, \mathrm{~F}_{\mathrm{N}} *\right)$ and $\left(\mathrm{M}^{*}\right.$, $\left.\mathrm{F}_{\mathrm{C}} *, \mathrm{~F}_{\mathrm{N}}{ }^{*} \pm 0.1\right)$, where point $\left(\mathrm{M}^{*}, \mathrm{~F}_{\mathrm{C}} *, \mathrm{~F}_{\mathrm{N}}{ }^{*}\right)$ indicates the central cell in the limit cycle. It seems that these cells are almost synchronized, and average output (represented a red line) has high amplitudes as well as single cells. Actually, these amplitudes are increased by this stimulus. Figure 3D (stimuli at CT22 with duration 15s) exhibits the similar dynamics. Although in Figure 3B the amplitudes are not increased by the stimulus (stimulus at CT12 with duration 10s), they at least maintain the original level. However, Figure 3C (stimulus at CT17.35 with duration 15s) exhibits the a totally different behavior. The average output (red line) totally loses the oscillation in the first 
day after the stimulus, and keeps a very low amplitude even 83 hours later. In this case, the two proposed mechanisms, amplitude loss in all cells and strong desynchronization in phases, are both present. To further investigate what kind of roles are they playing in singularity behavior, we compare Figure 3C with Figure 3E (CT17.35 with duration 12.5s) and 3F (CT17.35 with duration 17.5s). In 3E and 3F, cell amplitudes are weaken after the stimulus, but basically synchronized. Thus the average output slowly recovers along with the single cell amplitudes. However in 3C, due to the strong desynchronization, the average output is much lower than the single cell amplitudes. At CT100, the amplitudes of single cells are almost restored, while the average output is only $1 / 4$ of the original ones. Therefore, these two causes (amplitude loss and desynchronization) are all necessary for the fully singularity responses.

Next, we will explore the dynamical mechanism through the stability analysis.
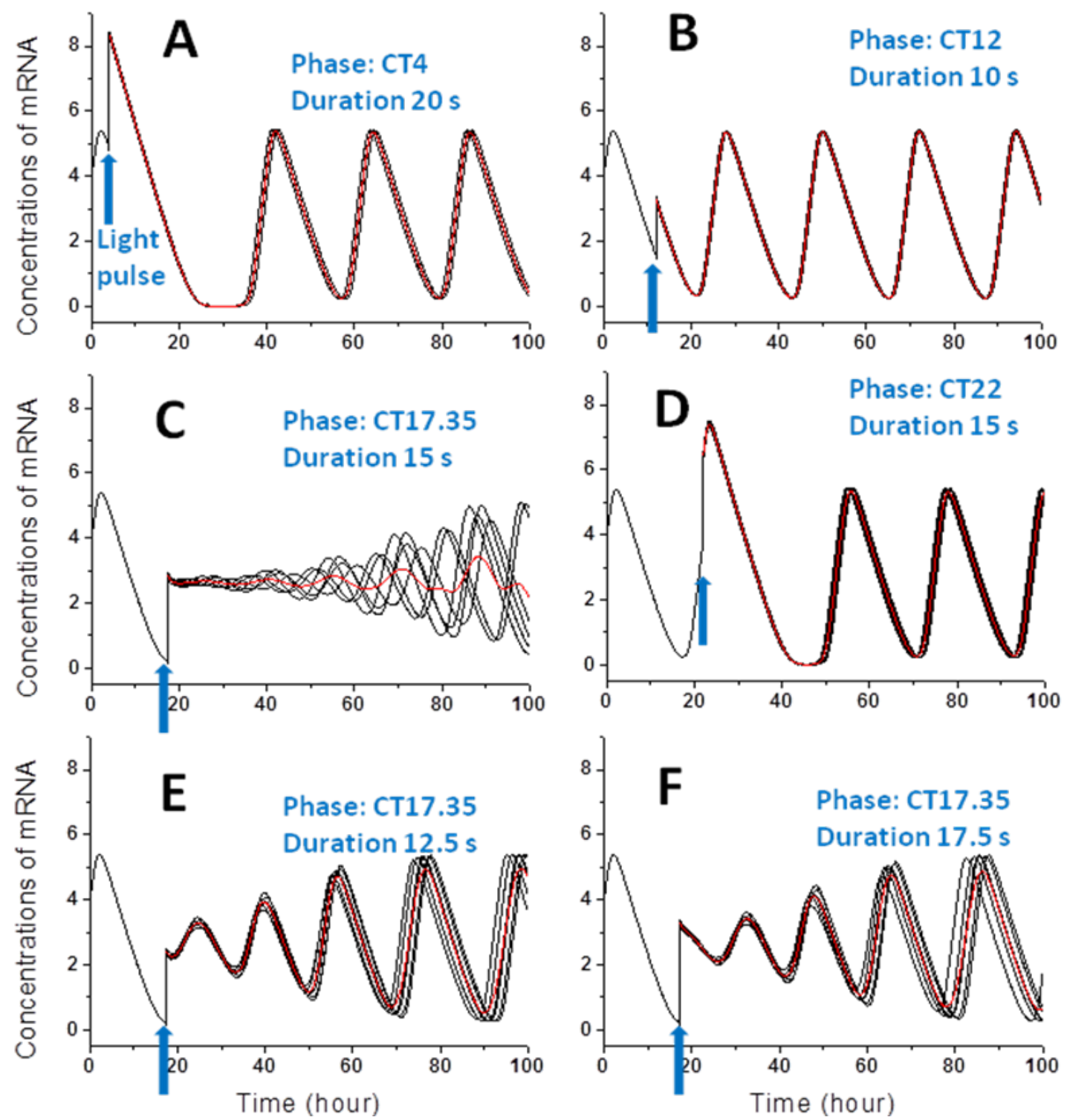

\subsection{The singularity dynamics in 3-D phase space}

Although an explicit expression cannot be obtained, system (1) possesses a unique steady state, defined by the $\frac{d M}{d t}=\frac{d F_{c}}{d t}=\frac{d F_{\mathbb{N}}}{d t}=0$. 
By a direct calculation of the first equation in system(1) we have:

$$
\mathrm{M}=\frac{\mathrm{k}_{\mathrm{m}}}{1-\frac{\mathrm{ws}}{\mathrm{vm}^{2}} \times \frac{\mathrm{K}_{1}^{\mathrm{n}}}{\mathrm{K}_{1}^{\mathrm{n}}+\mathrm{F}_{\mathrm{N}}^{\mathrm{n}}}}-\mathrm{k}_{\mathrm{m}}
$$

Solving the last equation of system (1), we have $F_{c}=\frac{k_{1}}{k_{2}} * F_{N}$. By substituting it to $\frac{d F_{c}}{d t}=0$, we get that

$$
\mathrm{M}=\frac{\mathrm{wd}}{\mathrm{ks}} * \frac{\frac{\mathrm{k}_{1}}{\mathrm{k}_{2}} ; F_{\mathrm{w}}}{\mathrm{K}_{\mathrm{d}}+\frac{\mathrm{k}_{1}}{\mathrm{k}_{2}} * F_{\mathrm{N}}}
$$

The steady states of system (1) must satisfy both eq(2) and eq(3). It's clear that in (2) M is a decreasing function of $F_{N}$, and in (3) $M$ is a increasing function of $F_{N}$. Hence, there is a only one solution satisfying both (2) and (3). Therefore, system (1) has a unique steady state.

The local stability of the unique steady state depends on parameters. So we use the parameters set from reference[18] to simulate the unique equilibrium point and its linear stability. Under this parameters set, the unique steady point $S^{*}=(2.63012,1.5482,1.29016)$, and it's Jacobian matrix is

$$
A=\left[\begin{array}{ccc}
-0.0258 & 0 & -0.9667 \\
0.5000 & -0.5515 & 0.6000 \\
0 & 0.5000 & -0.6000
\end{array}\right]
$$

The eigenvalues of A are $\lambda_{1}=-1.2783$ and $\lambda_{2,3}=0.0505 \pm 0.4326 \mathrm{i}$. Therefore, $\mathrm{S}^{*}$ has an one-dimensional stable manifold (red curve in Figure 4A and 4B) and a two-dimensional unstable manifold. Because the stable eigenvalue is 25 times higher of the unstable ones, the movement along the stable manifold is much faster than along the unstable manifold. 

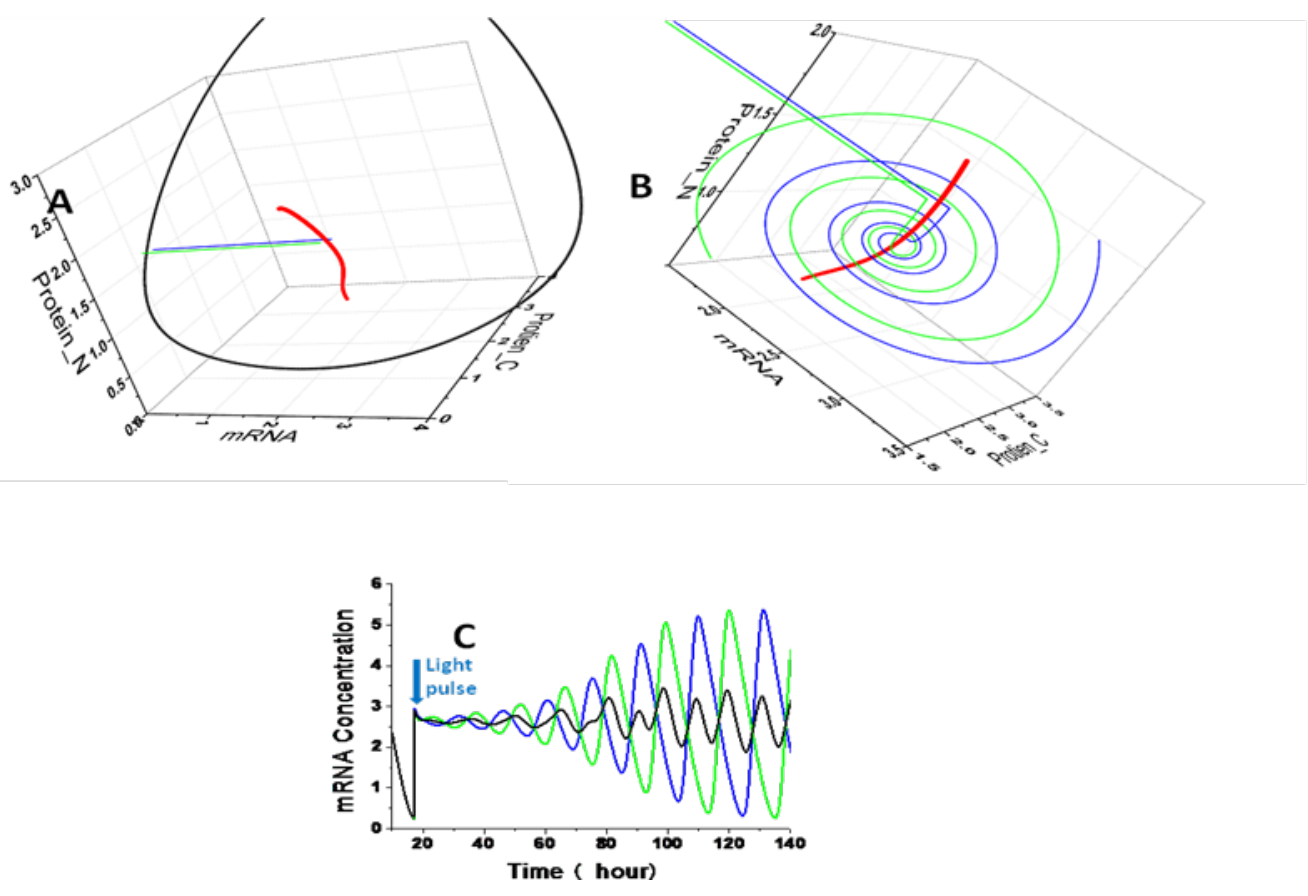

We then study what does the critical stimulus do in the 3-D phase diagram. Figure 4A shows the orbits (blue curve and green curve) of 2 points in the six-point-group during the light pulse stimulus. These 2 points are shifted from the neighborhood of the limit cycle to the neighborhood of the stable manifold (red curve). After the light pulse stopped, these orbits first go along with the stable manifold back to the neighborhood of the steady state. This is because that the stable eigenvalue is much higher of the unstable ones. In this stage, the amplitudes maintain in a extremely low level. Then these orbits go along the unstable manifold toward the stable limit cycle (Figure 4B). Since the real part of unstable eigenvalues is small, the amplitude recovery of cells is slow in this stage. Additionally, the critical stimulus also desynchronized these orbits by pushing them to the different sides of the stable manifold. Ideally, the "central cell" of this group was in the limit cycle before the stimulus, and the light pulse shifts the "central cell" into the stable manifold (shifted central cell denoted as SCC). Figure 4C shows the time course of two orbits and their average output (black) after the critical stimulus. In this situation, the six cells should be surrounding SCC, which is in the manifold. Hence, the phases of the cells could be separated by the stable manifold. Therefore, the desynchronization further lowers the group amplitude due to the neutralization among them.

Thus, the results suggest that the collaboration of the desynchronization and the loss of oscillation amplitude leads to the abolishment of the circadian oscillation. Furthermore, we found that the stable manifold of the unstable equilibrium point, instead of the steady state itself, plays a crucial role in circadian singularity behavior.

\section{Conclusion and Discussion}

In this paper, we study the dynamical mechanism of singularity behavior. In circadian clock, singularity behavior refers to a phenomenon by which the oscillation can be abolished after a 
proper stimulus (at an appropriate time with the right strength), such as a light or temperature change.

Previous studies suggested two alternative models to explain the mechanism: (1) desynchronization of the phases of oscillating cells in the cell population; (2) loss of oscillation amplitude in all cells by resetting the clock at or close to the steady state. We use a simple mathematical model to reveal the underlying mechanism. Our findings suggest that the critical singularity stimulus pushes the cells from the neighborhood of the limit cycle to the neighborhood of the stable manifold (instead of the neighborhood of the steady state, which was envisaged by early studies) to eliminate their amplitudes, and also desynchronize their phases. These two factors collaborate to abolish the circadian rhythmicities.

Although the synchronization is a core problem in the biological oscillations [19, 20], the previous mathematical model also suggested a hard excitation explanation for singularity behavior: suppression occurs in conditions where a stable steady state coexists with limit cycle oscillations, and the pulse-induced transition of pacemaker cells from a stable oscillatory regime into a stable steady state [14]. In our model, because the steady state is unstable, the oscillations progressively resume, but it takes a large number of cycles to return to the amplitude of oscillations prior to perturbation by the light pulse. Moreover, the global rhythm is more attenuated than the rhythms in individual cells. Therefore, the phenomenon does correspond to suppression of the circadian rhythms even though such suppression is transient in any given cell. In view that hard excitation is a phenomenon that occurs in particular conditions, and not in all models for circadian oscillations, the present results are likely to be more generic for the suppression of circadian rhythmicity by transient perturbations.

When circadian rhythmicity is suppressed by a single transient perturbation, it can often be restored by a second pulse. This phenomenon, observed in some organisms, was modeled theoretically by Leloup and Goldbeter[14] in the case of hard excitation : the first pulse brings the oscillating system to the singularity, which in that case is a stable steady state, and the second pulse switches the system back to the limit cycle. In our model, a second pulse of light can also accelerate the return to the oscillations (data does not show) by resychronizing the cells and restoring the amplitude of individual cells.

In this study we employ a very simple model for Neurospora to investigate the basic mechanism of singularity behavior. Actually in higher organisms, the circadian clock involves more complicated elements, which may Influence the occurrence of singularity behavior. For example, in mice[11] it takes several hours (compare with 15 seconds for Neurospora) to achieve abolishment of the oscillation. Since the stimulus duration is in the same time scale of the oscillation period, one has to take into account these two factors simultaneous. Furthermore, higher organisms can effectively be resistant to the outside stimuli. In human[10], mice[11] and hamster[13], it usually takes more than one stimulus to induce arrhythmicity. Additionally, the recovery velocity of the amplitude in single cells, and the ability of resynchronization in population might be improved in higher organisms by natural selection. Therefore, the effects of these elements, including the coordination of exogenous stimulus and endogenous clock, the resistance ability (equivalent to limitations of the stimulus), recovery speed of single oscillator, and the competition between synchronization ability and the noise intensity, are worth of exploration in future work. However, we believe that moving the orbits to the neighborhood of a $\mathrm{n}$-2 dimensional stable manifold of the unstable steady state (in an n- dimensional clock system) is 
a key requirement for inducing singularity behavior.

\section{Acknowledgments}

This work is supported by National Science Foundation of China (No. 61271358), and the Priority Academic Program of Jiangsu Higher Education Institutions.

\section{References}

1 Lowrey, P.L., and Takahashi, J.S.: 'Mammalian circadian biology: elucidating genome-wide levels of temporal organization', Annu Rev Genomics Hum Genet, 2004, 5, pp. 407-441

2 Mohawk, J.A., and Takahashi, J.S.: 'Cell autonomy and synchrony of suprachiasmatic nucleus circadian oscillators', Trends in neurosciences, 2011, 34, (7), pp. 349-358

3 Goldbeter, A.: 'Biochemical Oscillations and Cellular Rhythms: The molecular bases of periodic and chaotic behaviour' (Cambridge University Press, 1996. 1996)

4 Zhang, E.E., and Kay, S.A.: 'Clocks not winding down: unravelling circadian networks', Nature reviews. Molecular cell biology, 2010, 11, (11), pp. 764-776

5 Yan, J., Shi, G., Zhang, Z., Wu, X., Liu, Z., Xing, L., Qu, Z., Dong, Z., Yang, L., and Xu, Y.: 'An intensity ratio of interlocking loops determines circadian period length', Nucleic Acids Res, 2014, 42, (16), pp. 10278-10287

6 Goldbeter, A.: 'A model for circadian oscillations in the Drosophila period protein (PER)', Proc Biol Sci, 1995, 261, (1362), pp. 319-324

7 Ouyang, Y., Andersson, C.R., Kondo, T., Golden, S.S., and Johnson, C.H.: 'Resonating circadian clocks enhance fitness in cyanobacteria', Proceedings of the National Academy of Sciences of the United States of America, 1998, 95, (15), pp. 8660-8664

8 Scheer, F.A., Hilton, M.F., Mantzoros, C.S., and Shea, S.A.: 'Adverse metabolic and cardiovascular consequences of circadian misalignment', Proceedings of the National Academy of Sciences of the United States of America, 2009, 106, (11), pp. 4453-4458

9 Gu, X., Xing, L., Shi, G., Liu, Z., Wang, X., Qu, Z., Wu, X., Dong, Z., Gao, X., Liu, G., Yang, L., and $\mathrm{Xu}$, Y.: 'The circadian mutation PER2(S662G) is linked to cell cycle progression and tumorigenesis', Cell Death Differ, 2012, 19, (3), pp. 397-405

10 Jewett, M.E., Kronauer, R.E., and Czeisler, C.A.: 'Light-induced suppression of endogenous circadian amplitude in humans', Nature, 1991, 350, (6313), pp. 59-62

11 Ukai, H., Kobayashi, T.J., Nagano, M., Masumoto, K.H., Sujino, M., Kondo, T., Yagita, K., Shigeyoshi, Y., and Ueda, H.R.: 'Melanopsin-dependent photo-perturbation reveals desynchronization underlying the singularity of mammalian circadian clocks', Nat Cell Biol, 2007, 9, (11), pp. 1327-1334 12 Huang, G., Wang, L., and Liu, Y.: 'Molecular mechanism of suppression of circadian rhythms by a critical stimulus', The EMBO journal, 2006, 25, (22), pp. 5349-5357

13 Fernandez, F., Lu, D., Ha, P., Costacurta, P., Chavez, R., Heller, H.C., and Ruby, N.F.: 'Circadian rhythm. Dysrhythmia in the suprachiasmatic nucleus inhibits memory processing', Science, 2014, 346, (6211), pp. 854-857

14 Leloup, J.C., and Goldbeter, A.: 'A molecular explanation for the long-term suppression of circadian rhythms by a single light pulse', American journal of physiology. Regulatory, integrative and comparative physiology, 2001, 280, (4), pp. R1206-1212

15 Winfree, A.T.: ‘The geometry of biological time’ (Springer Verlag, 1980. 1980) 
16 Johnson, C.H., Elliott, J.A., and Foster, R.: ‘Entrainment of circadian programs’, Chronobiology international, 2003, 20, (5), pp. 741-774

17 Tan, Y., Dragovic, Z., Roenneberg, T., and Merrow, M.: 'Entrainment dissociates transcription and translation of a circadian clock gene in neurospora’, Current biology : CB, 2004, 14, (5), pp. 433-438

18 Gonze, D., Leloup, J.C., and Goldbeter, A.: 'Theoretical models for circadian rhythms in Neurospora and Drosophila’, C R Acad Sci III, 2000, 323, (1), pp. 57-67

19 Acebrón, J.A., Bonilla, L.L., Pérez Vicente, C.J., Ritort, F., and Spigler, R.: 'The Kuramoto model: A simple paradigm for synchronization phenomena', Reviews of Modern Physics, 2005, 77, (1), pp.

137-185

20 Glass, L.: 'Synchronization and rhythmic processes in physiology', Nature, 2001, 410, (6825), pp. $277-284$ 


\section{Figure Legends}

Figure 1. Mathematical model of Neurospora circadian system. (A) is the scheme of the model for circadian oscillations in Neurospora. The model is based on the negative feedback exerted by the protein FRQ on the transcription of the frq gene; the rate of gene expression is enhanced by light. This model includes gene transcription in the nucleus, accumulation of the corresponding mRNA in the cytosol with the associated protein synthesis, protein transport into and out of the nucleus, and regulation of gene expression by the nuclear form of FRQ. The panels (B) and (C) show the numerical simulation results for above model. This model generates autonomous oscillations in constant darkness (B), and a light pulse can directly switch the frq mRNA and indirectly increase the concentrations of FRG proteins. Parameter values are: $v \mathrm{~s}=1.6 \mathrm{nMh}^{-1}, v \mathrm{~m}=0.505 \mathrm{nMh}^{-1}, v \mathrm{~d}$ $=1.4 \mathrm{nMh}^{-1}, k \mathrm{~s}=0.5 \mathrm{~h}^{-1}, k 1=0.5 \mathrm{~h}^{-1}, k 2=0.6 \mathrm{~h}^{-1}, \mathrm{Km}=0.5 \mathrm{nM}, K \mathrm{I}=1 \mathrm{nM}, K \mathrm{~d}=0.13 \mathrm{nM}, n=4$. The light pulse item $\mathrm{I}_{\text {light }}=630 \mathrm{nMh}^{-1}$ when light is on. The initial values are: $\mathrm{M}=3.9796, \mathrm{~F}_{\mathrm{C}}=0.8867, \mathrm{~F}_{\mathrm{N}}=0.4771$, which is a point in the limit cycle.

Figure 2. Finding singularity behavior. (A) is the illustration of the cell selection in the group and the effect of the light-pulse stimulus in the 3-D phase space. The left panel of (B) shows the hot map result for the amplitude level in the next 24 hour after the stimuli. The stimuli happen in the phase of CT0, CT2, CT4, ... CT22, with the light pulse durations: 10s, 15s and 20s. The panel right to it is the result of a refined scan: stimulus phase from CT14 to CT CT18, increasing by 0.4 hour; light pulse durations are $12.5 \mathrm{~s}, 15 \mathrm{~s}$ and $17.5 \mathrm{~s}$. The right panel of (B) is a color bar indicating the amplitude level from 0 (blue) to 10 (yellow) in arbitrary unit.

Figure 3. Detail dynamics after a light pulse stimuli. The responses of six slightly different cells (black lines) and their average outputs (red line) are shown in six different cases. The stimulus phases are indicated by the blue arrows. (A), (B) and (D) display non-singularity responses. (C) exhibits the fully singularity behavior, when the amplitude loss in all cells and the strong desynchronization in phases are all in present. (E) and (D) show the quasi-singularity responses when the strong desynchronization is absent.

Figure 4. Singularity mechanism in 3-D phase diagram. (A) shows the orbits (blue curve and green curve) of 2 points in the six-point-group during the light pulse stimulus. The critical stimulus shifts the points from the neighborhood of the stable limit cycle (black curve) to the neighborhood of the stable manifold (red curve) of the equilibrium point. (B) shows the detailed dynamics of two orbits after the critical stimulus in the phase space. Note that the view angles are different in two panels. (C) shows the time course of two orbits and their average output after the critical stimulus. 